\title{
Fibroblast growth factor 2 induces mesenchymal stem cells to differentiate into tenocytes through the MAPK pathway
}

\author{
TAO-YI CAI ${ }^{*}$, WEI ZHU* , XIONG-SHENG CHEN, SHENG-YUAN ZHOU, LIAN-SHUN JIA and YAN-QING SUN \\ Department of Orthopedic Surgery, Changzheng Hospital, Second Military Medical University, Shanghai 200003, P.R. China
}

Received December 12, 2012; Accepted August 8, 2013

DOI: $10.3892 / \mathrm{mmr} .2013 .1668$

\begin{abstract}
Mesenchymal stem cells (MSCs) differentiate into numerous different cell types and thus have therapeutic potential for tissue engineering, anti-inflammatory and immunomodulatory purposes. FGF2 may affect the biological behavior of MSCs. MSCs were transduced with either adenovirus-null vector/green fluorescent protein (GFP) or a vector encoding for the overexpression of FGF2/GFP. The expression of FGF2 was demonstrated to be significantly higher in MSC (FGF2) compared with MSC (vector) by qPCR and western blot analysis. In order to investigate the function of FGF2 in MSCs over time, it was observed that FGF2 stimulates cell proliferation and induces cell differentiation by activating the mitogen-activated protein kinase (MAPK) signaling pathway. Following blockade of the FGF2-induced activation of the extracellular signal-regulated kinase pathway by overexpression of sprouty isoforms, the marker of differentiation markedly decreased. Altogether, the results demonstrated a novel cell biological mechanism that FGF2 differentiates into tenocytes and the MAPK pathway is key in differentiation.
\end{abstract}

\section{Introduction}

Tendons are connective tissues that join muscle to bone. Tendon injuries are painful and widely distributed clinical problems in society. While the healing of such disorders leads to costly medical expenses, the original biological properties of the tissue do not return to normal (1-3). Although there have been advances in the development and maintenance of tendon and ligament tissue, and on cellular and gene therapy

Correspondence to: Professor Xiong-Sheng Chen, Department of Orthopedics Surgery, Shanghai Changzheng Hospital, Second Military Medical University, 415 Fengyang Road, Shanghai 200003, P.R. China

E-mail: chenxiongsheng@vip.sohu.com

*Contributed equally

Key words: mesenchymal stem cells, fibroblast growth factor 2, tendon, extracellular signal-regulated kinase approaches for repair, tissue engineering remains far from being capable of producing the ideal bioscaffold to replace, repair or regenerate injured ligaments $(4,5)$. However, mesenchymal stem cells (MSCs) have been considered as a source of cells for use in ligament regeneration.

MSCs are a pluripotent cell population capable of differentiating into a variety of cell types, and were originally isolated from the bone marrow (BM) (6). MSCs have been considered to be promising tools for regeneration and cancer therapy in several preclinical and clinical trials due to their plasticity (7-9). Several studies support the hypothesis that MSCs differentiate into multiple cell lineages, including osteoblasts, chondrocytes, adipocytes, smooth muscle cells, skeletal and cardiac myocytes, endothelial cells and neurons $(10,11)$. Growth of MSCs throughout life involves proliferation of epithelial cells and their subsequent differentiation into multiple cell lineages. An increasing number of studies have demonstrated the involvement of fibroblast growth factor 2 (FGF2) in regulating cell growth and differentiation; however, its effect on MSCs appears complex and remains unclear.

FGF2 is a member of the FGF family, which is synthesized as a 155 amino acid precursor and is subsequently processed into a mature form consisting of 146 amino acid residues (12). FGF2 mediates cellular signal transduction through binding to fibroblast growth factor tyrosine kinase receptors (FGFRs) and downstream signaling molecules, such as phosphatidylinositide 3-kinase (PI3K)-Akt and Ras-Raf-mitogen activated protein kinase (MAPK) (13). However, FGF2 exhibits bidirectional effects on growth and differentiation of MSCs. FGF2 has been shown to stimulate growth and preserve the differentiation potential of MSCs during long-term culture expansion in vitro by inducing cell motility, the expression of vimentin (VIM) and $\alpha$-smooth muscle actin (SMA) (14-19). FGF2 has also been demonstrated to stimulate chondrogenic and adipogenic differentiation of human $(20,21)$ and rat (22) MSCs, respectively. By contrast, recent studies have shown that the inhibition of mouse MSC differentiation by FGF2 is strongly correlated with the upregulation of Twist2 and Spry4, and the suppression of extracellular signal-regulated kinase (ERK)1/2 activation (23). Thus, a number of intracellular signaling cascades may be involved, in particular the MAPK pathway, in which sequential phosphorylation of a series of protein kinases ultimately activates ERK to control a variety of downstream responses, including gene transcription. To 
investigate the molecular mechanisms underlying the involvement of FGF2 in MSCs is currently a predominant aim in biomedical research.

In the present study, it was hypothesized that the differentiation induced by FGF2 may be mediated by alterations in the levels of mRNA and proteins with known specific extracellular matrix proteins and cytoskeletal elements. The effects of the overexpression of FGF2 on collagen I, collagen III, scleraxis, fibronectin and $\alpha$-SMA expression were determined in vitro, and the involvement of the MAPK signaling pathway was investigated in MSC differentiation.

\section{Materials and methods}

Isolation and expansion of human MSCs. Human MSCs were isolated from the iliac crest obtained from four human donors due to vertebral fractures. The donor population was reasonably homogenous (age, 25-47 years, equal numbers of males and females). All procedures were approved by the ethics committee of the Second Militarty Medical University, Shanghai, China, and informed consent was obtained from all donors. MSCs were isolated with a density gradient and resuspended in complete culture medium. Human MSCs were cultured in Dulbecco's modified Eagle's medium-high glucose containing $10 \%$ fetal calf serum, $50 \mu \mathrm{M} 2-\mathrm{ME}$ (Sigma-Aldrich), penicillin/streptomycin (100 U/ml/100 $\mu \mathrm{g} / \mathrm{ml}$, HyClone) at $37^{\circ} \mathrm{C}$ in a humid atmosphere with $5 \% \mathrm{CO}_{2} . \mathrm{MSCs}$ were cultured as adherent cells, non-adherent cells were removed by medium change after three days. The medium was replaced three days following plating and then every three days subsequent to that. Cells from each donor were cultured separately. Cells were passaged at 70-80\% confluence and passages 3-8 were used for experiments.

RNA isolation and $q P C R$. Total RNA was extracted from monolayer cells by TRIzol (Invitrogen Life Technologies, Carlsbad, CA, USA). RNA quality was assessed by the Blue Pippin automated electrophoresis system (Sage Science, Inc., Beverly, MA, USA) using the Experion RNA StdSens Analysis kit (Bio-Rad, Hercules, CA, USA). cDNA was prepared using the PrimeScript II First Strand cDNA Synthesis kit (Takara Bio Inc., Shiga, Japan) according to the manufacturer's instructions, and $1 \mu \mathrm{l}$ 5X diluted cDNA was used for further gene amplification. qPCR was performed by FastStart Universal SYBR-Green Master (Rox; Roche Dianostics, Mannheim, Germany). PCR was performed in a Light Cycler real-time PCR machine (Bio-Rad) with the following thermal profile: $2 \mathrm{~min}$ at $95^{\circ} \mathrm{C}$ followed by 40 cycles of denaturation at $95^{\circ} \mathrm{C}$ for $30 \mathrm{sec}$, annealing at $58^{\circ} \mathrm{C}$ for $30 \mathrm{sec}$, extension at $72^{\circ} \mathrm{C}$ for $1 \mathrm{~min}$ and a final extension at $72^{\circ} \mathrm{C}$ for $3 \mathrm{~min}$. The primers used were as follows: Forward: 5'-GGTGATGGTGGGAATGGG-3', and reverse: 5'-GCAGGGTGGGATGCTCTT-3' for $\alpha$-SMA; forward: 5'-TTCCTGCGCCTGATGTCC-3' and reverse 5'-GGTTCAGTTTGGGTTGCTTGT-3' for collagen I; forward: 5'-TGAAAGGACACAGAGGCTTCG-3' and reverse: 5'-GCACCATTCTTACCAGGCTC-3' for collagen III; forward: 5'-TTCCTGCGCCTGATGTCC-3' and reverse: 5'-GGTTCAGTTTGGGTTGCTTGT-3' for FGF2; forward: 5'-TCAGAAGAGCGAGCCCCT-3' and reverse: 5'-GGGGTCTTTTGAACTGTGGA-3' for fibronectin; forward: 5'-CCAGGCAAAGCAGGAGTC-3' and reverse: 5'-GGGTATCAACCAGAGGGAGT-3' for vimentin; forward: 5'-CATCTCGCACCTGGGCAA-3' and reverse: 5'-CTGTTTGGGCTGGGTGTTC-3' for scleraxis; and forward: 5'-GGGAAACTGTGGCGTGAT-3' and reverse 5'-GTGGTCGTTGAGGGCAAT-3' for glyceraldehyde 3-phosphate dehydrogenase (GAPDH). All primers were synthesized by Takara. Data was analyzed using Bio-Rad iQ5 software. Expression of endothelial genes was calculated relative to GAPDH levels by the comparative $\triangle \mathrm{CT}$ method.

Western blot analysis. The cells were harvested and lysed in RIPA buffer on ice. The cell lysates were heated at $100^{\circ} \mathrm{C}$ for $5 \mathrm{~min}$ and centrifuged at $16,000 \mathrm{x} \mathrm{g}$ for $10 \mathrm{~min}$. The supernatant was collected and the protein concentration was determined by the Pierce Bicinchoninic Acid Protein Assay kit (Thermo Scientific, Waltham, MA, USA). Equivalent quantities of protein $(40 \mu \mathrm{g})$ from each sample were loaded and run on sodium dodecyl sulfate-polyacrylamide gel electrophoresis gels (Bio-Rad) and transferred onto nitrocellulose membranes (Bio-Rad). Subsequent to blocking the membranes with $2 \%$ bovine serum albumin in Tris-buffered saline with $0.1 \%$ Tween 20 at room temperature for $2 \mathrm{~h}$, the membranes were incubated with primary antibodies in a 1:1,000 dilution at $4^{\circ} \mathrm{C}$ overnight, washed with phosphate-buffered saline with Tween 20 (PBST), and incubated with horseradish peroxidase-conjugated secondary antibodies for $1 \mathrm{~h}$ at room temperature. Antibodies used were as follows: Anti-FGF2 (catalog no. ab106245; Abcam, Cambridge, UK), phospho-p42/44MAPK (Thr-202/Tyr-204; catalog no. 9101; Cell Signaling Technology, Inc., Danvers, MA, USA), p42/44MAPK (catalog no. 9102; Cell Signaling Technology, Inc.), collagen I (catalog no. ab21285; Abcam), collagen III (catalog no. ab7778, Abcam), scleraxis (catalog no. sc-87425; Santa Cruz Biotechnology Inc., Santa Cruz, CA, USA), fibronectin (catalog no. AB2033; Millipore, Billerica, MA, USA) and $\alpha$-smooth muscle actin (catalog no. PA5-19465; Thermo Scientific Pierce, Rockford, IL, USA). Subsequent to washing with PBST, the immunoblots were visualized by chemiluminescence using an enhanced chemiluminescent western blot analysis substrate (Millipore). GAPDH was used to ensure equal protein loading.

Construction plasmids and recombinant adenoviruses. Adenovirus was generated using the pAdTrack/pAdEasy system. Briefly, full-length FGF2 cDNA was subcloned into pAdTrack as described previously (24). The pAdTrack plasmid was cotransfected with pAdEasy into DH5 $\alpha$ cells to generate recombinant plasmids. Recombinants $(10 \mu \mathrm{g})$ were linearized with $P a c$ I restriction enzyme (New England Biolabs, Ipswich, MA, USA) and transfected into 293A packaging cells (from laboratory stock). Recombinant adenoviruses were prepared, purified and titered as described previously (25). MSCs were infected with vehicle adenovirus or Ad-FGF2 at $2 \times 10^{7}$ multiplicity of infection (MOI)/ml for $24 \mathrm{~h}$. Adenoviral infection efficiency was assessed by western blot analysis. The cDNAs encoding full open reading frames of murine Spry1 and Spry4 were amplified and subcloned into pcDNA3.1(+) (Invitrogen Life Technologies) with the BamHI and EcoRI sites. The 
A

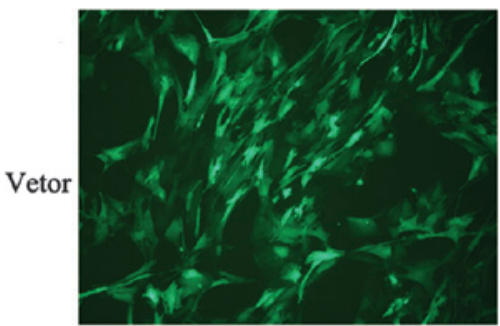

B

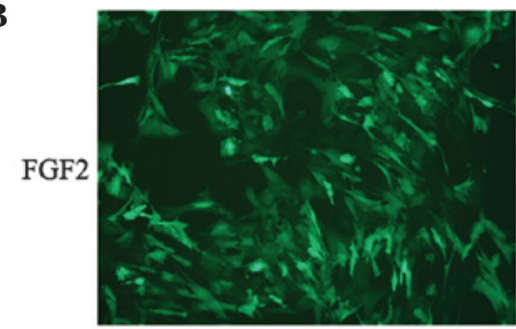

C

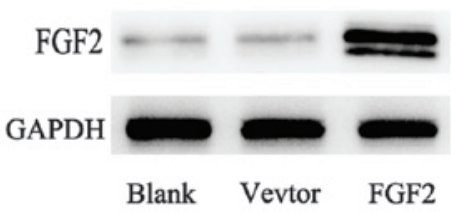

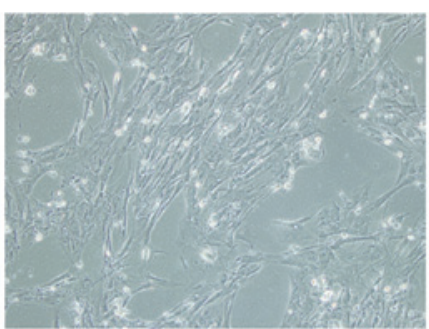

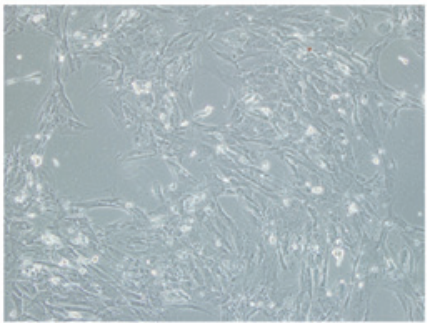

D

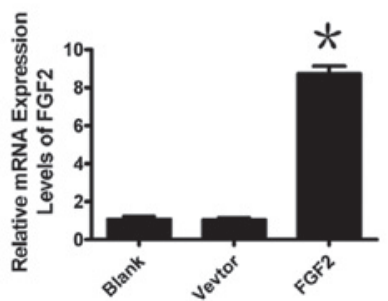

Figure 1. Ectopic FGF2 expression in MSCs. Bone marrow MSCs were infected with Ad-FGF2 or Ad-GFP (MOI=40), efficient transduction was calculated by counting the number of green fluorescent cells in a population of 1000 cells in four randomly selected areas per dish. (A) Representative photomicrographs of FGF2-GFP-transfected cells. (B) Representative photomicrographs of GFP-transfected cells. (C and D) mRNA and protein expression of FGF2 was evaluated by western blot analysis and qPCR, and GAPDH served as an internal control. " $\mathrm{P}<0.05$ versus control. MSCs, mesenchymal stem cells; GFP, green fluorescent protein; GAPDH, glyceraldehyde 3-phosphate dehydrogenase; FGF2, fibroblast growth factor 2.

corresponding segments were amplified using PCR with the following primers: Forward: 5'-AAAGGATCCATGGATTCC CCAAGTCAGC-3' and reverse: 5'-AAAGAATTCTCATGA CAGTTTGCCCTGAG-3' for Spry1; and forward: 5'-AAA GGATCCATGGAGCCCCCGGTTCCA-3' and reverse, 5'-AAAGAATTCTCAGAAAGGCTTGTCAGACCTGC-3' for Spry4.

For transient co-transfection of Spryl and Spry4, MSC cells were seeded onto $60 \mathrm{~mm}$ dishes and transfected with $4 \mu \mathrm{g} / \mathrm{dish}$ each plasmid or a control vector using Lipofectamine ${ }^{\mathrm{TM}} 2000$ reagent (Invitrogen Life Technologies) according to the manufacturer's instructions.

Cell proliferation assay. A cell proliferation assay was performed using tetrazolium compound based CellTiter $96^{\circledR}$ Aqueous One Solution Cell Proliferation (MTS) assay (Promega Corporation, Madison, WI, USA). Cells of each MSC clone were seeded into wells of a 96-well plate at a density of $4 \times 10^{3}$. After 24,48 , and $72 \mathrm{~h}$ of culture, an MTS assay was performed according to the manufacturer's instructions. Each experiment was performed in triplicate and repeated 3 times $(n=3)$.

Statistical analysis. Results are expressed as the mean \pm SD. Significance was analyzed with a two-tailed unpaired t-test using GraphPad Prism 5 Demo software for Windows (GraphPad Software, San Diego, CA, USA). P $<0.05$ was considered to indicate a statistically significant difference.

\section{Results}

Expression of FGF2 in MSCs by adenoviral vectors. To gain an understanding of the involvement of FGF2 in MSCs, MSCs were infected with a replication deficient Ad5 vector encoding FGF2 and GFP, or the control adenoviral vector, Ad-GFP. Green fluorescence identifies adenovirus-infected cells following infection with FGF2 or GFP (Fig. 1A and B). A high infection efficiency was observed when the MOI was 40 and the expression of mRNA and protein of FGF2 were verified by qPCR and western blot analysis (Fig. 1C and D). The mRNA and protein expression of FGF2 were markedly increased following treatment of MSCs with Ad-FGF2, whereas treatment with the negative control did not result in any change in FGF2 expression.

Overexpression of FGF2 induces tendon marker expression. To demonstrate the therapeutic potential of FGF2 in tendon injury, the expression of several important genes involved in the differentiation of MSCs were analyzed on days 4, 7 or 14. The mRNA of FGF2 was significantly increased in 
A

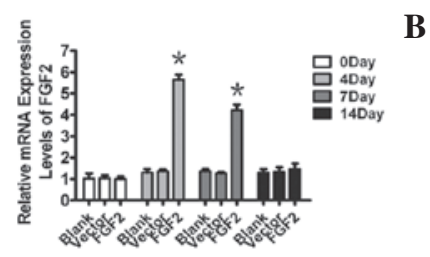

D

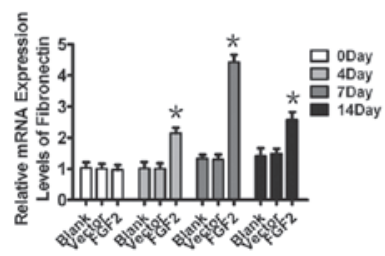

G

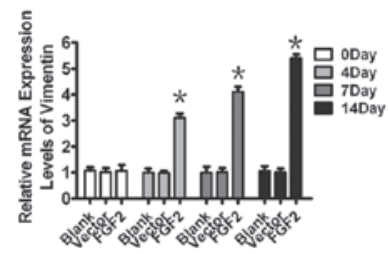

B

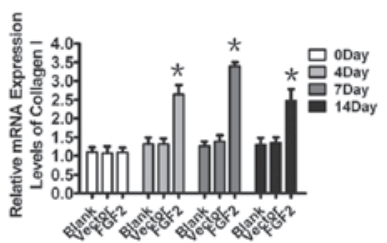

$\mathbf{E}$

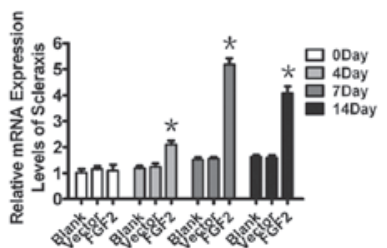

C

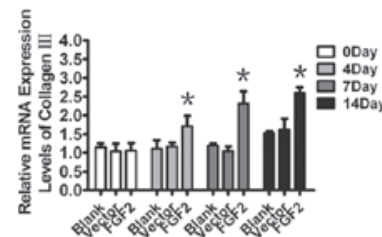

$\mathbf{F}$

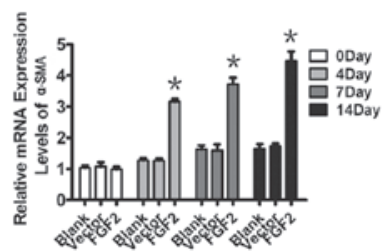

H

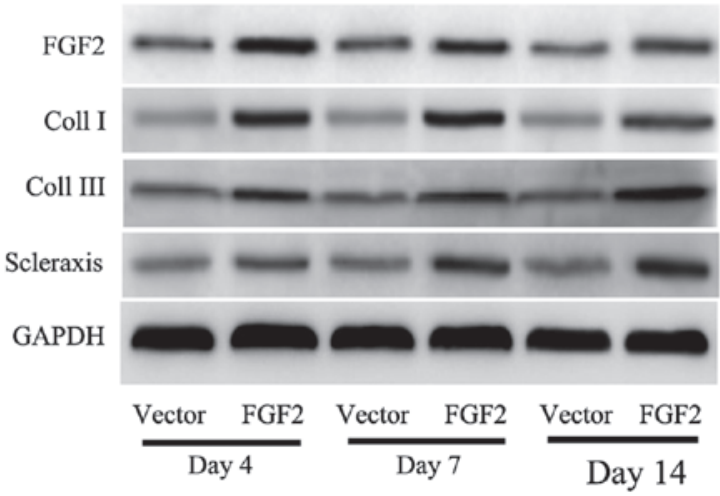

Figure 2. Relative mRNA expressions of key cytokines and growth factors involved in the differentiation of mesenchymal stem cells. (A-G) Relative mRNA expression of FGF2, Coll I, Coll III, fibronectin, scleraxis, $\alpha$-SMA and vimentin was measured by qPCR and corrected with GAPDH levels. Data are expressed as the mean \pm SE; " $\mathrm{P}<0.05$ vs control. (H) The detection of FGF2, Coll I, Coll III and scleraxis was confirmed by western blot analysis on days 4,7 and 14 . FGF2, fibroblast growth factor 2; SMA, smooth muscle actin; GAPDH, glyceraldehyde 3-phosphate dehydrogenase; Coll, collagen.

A

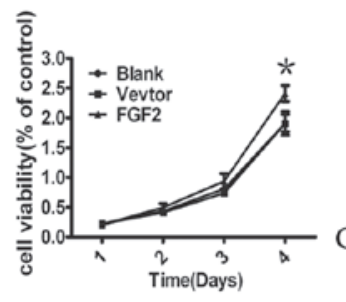

B

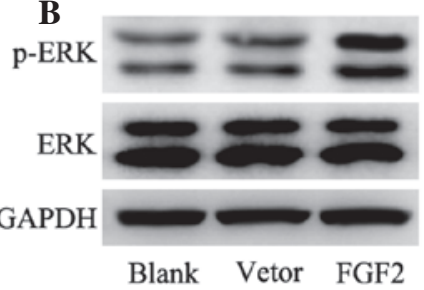

Figure 3. The effect of FGF2 on cell proliferation and the mitogen-activated protein kinase pathway. (A) Effect of FGF2 expression on proliferation of MSCs. An MTT cell viability assay was performed at 24, 48, 72 and $96 \mathrm{~h}$ following infection of MSCs with Ad-FGF2 or Ad-GFP. (B) The levels of p-ERK and ERK were assessed using western blot analysis in MSCs infected with Ad-FGF2 or Ad-GFP. FGF2, fibroblast growth factor 2; MSCs, mesenchymal stem cells; GFP, green fluorescent protein; p-ERK, phosphorylated extracellular signal-regulated kinase.

FGF2-treated MSCs compared with the control on days 4 and 7 (Fig. 2A). As expected, exposing MSCs to the combination of adenoviral vectors encoding FGF2 resulted in the upregulation of the mRNA of molecular markers, including collagen I, collagen III, fibronectin, scleraxis, $\alpha$-SMA, and VMT on days 4, 7 and 14 (Fig. 2B-G). To confirm the change of the corresponding genes, their expression was detected by western blot analysis (Fig. 2H). These characteristics indicated that FGF2 induced MSCs to differentiate into tenocytes.
A
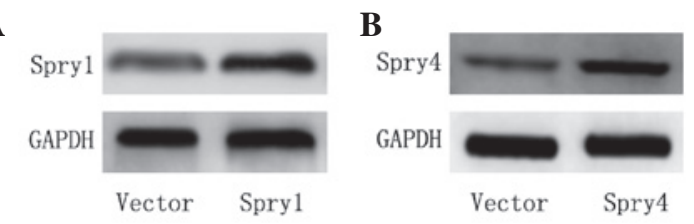

C

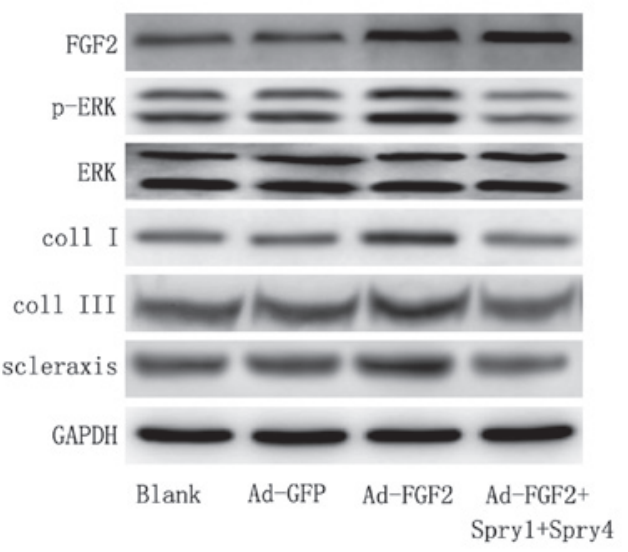

Figure 4. Differentiation of mesenchymal stem cells by FGF2 was suppressed through the mitogen-activated protein kinase pathway. (A) Cell lysates were collected and efficacy of overexpression was determined by sodium dodecyl sulfate-polyacrylamide gel electrophoresis and immunoblot analysis with antibodies to Spry1 and 4. (B) Expression of FGF2, p-ERK, ERK, Coll I, Coll III and scleraxis were analyzed by western blot analysis. GAPDH served as the loading control. FGF2, fibroblast growth factor 2; p-ERK, phosphorylated extracellular signal-regulated kinase; Coll, collagen; GAPDH, glyceraldehyde 3-phosphate dehydrogenase. 
FGF2 promotes cell growth and activation of MAPK signaling. To gain further insight into the biological effects of FGF2 on MSCs, cell growth was analyzed and it was identified that ectopic expression of FGF2 resulted in enhanced proliferation compared with control cells (Fig. 3A). To investigate the signaling pathways regulating the effects of FGF2, the MAPK pathway, a key cellular signal activated in pluripotent cells was analyzed (26). Consistent with these findings, ERK phosphorylation was upregulated in MSCs infected by Ad-FGF2 (Fig. 3B). These data suggest that the overexpression of FGF2 in MSCs resulted in cell proliferation, which appeared to be via the MAPK pathway.

Downstream signaling from FGF2 is ERK1/2 dependent. To further validate whether the activation of ERK was required in MSCs for differentiation into tenocytes, Spry1 and Spry4 were co-transfected, which have been shown to be efficient in the suppression of FGF2 induced ERK activation (27). The protein expression of Spry1 and Spry4 was significantly increased compared with control vector following ectopic expression of Spry1 and Spry4 (Fig. 4A and B). In addition, overexpression of Spryl and Spry4 inhibited the activation of ERK (Fig. 4C). Furthermore, the expression of Spry1 and Spry4 protein prevented the induction of collagen I, collagen III and scleraxis expression relative to that in untransfected cells (Fig. 4C). Overall, data suggest that the activation of MAPK is involved in the differentiation of MSCs by FGF2.

\section{Discussion}

BM MSCs have recently received widespread attention due to their capacities for multilineage differentiation and proliferation (6). To the best of our knowledge, the present study showed for the first time, that MAPK was important in the differentiation of MSCs induced by FGF2. The results indicated that FGF2 is able to promote the differentiation of MSCs into tendons by activating the MAPK pathway, which regulates the expression of molecular biomarkers of tenocytes.

To understand the molecular basis by which FGF2 determines the differentiation of MSCs, MSCs were maintained to high passage in vitro and retained their proliferative and multipotent abilities. The MSCs were obtained from human donors and cultured without the loss of endothelial potential normally observed in cells expanded up to such a level. Previous studies have shown that FGF2 exhibited bidirectional effects on the growth and differentiation of MSCs $(14,18,20,23)$. Expression of FGF2, which is an important tenocyte cytokine induced tendon matrix protein type I collagen, type III collagen and scleraxis. This result is consistent with studies showing that FGF2 is important in stimulating cell proliferation and differentiation (4).

To further investigate the downstream signaling which is required in MSC differentiation, it was hypothesized that ERK signaling is essential for tendon differentiation. Data demonstrated that phosphorylation of ERK1/2 is strongly upregulated in MSCs following overexpression of FGF2 and promoted cell growth. Recent studies by Ozaki et al (27) demonstrated that co-expression of Sprouty1 and Sprouty4 efficiently suppressed the FGF2-induced ERK activation. Sprouty1 and Sprouty4 were then co-transfected, which efficiently inhibited the ERK activation induced by FGF2, while the increase of collagen I, collagen III and scleraxis induced by FGF2 was inhibited. These results provided key insights in the involvement of MAPK signaling in FGF2-induced differentiation.

In conclusion, the results indicated that overexpression of FGF2 by adenovirus stimulates proliferation and expression of extracellular matrix proteins, which are required for the tissue engineering of tendons. In addition, it appears to differentiate MSCs into a more fibroblast-like cell type. It was also observed that FGF2 stimulated the activation of the phosphorylation of ERK1/2 leading to the upregulation of essential extracellular matrix molecules and cytoskeletal elements for ligaments and tendons. It may be useful to determine whether FGF signaling in MSCs is the same in vivo, as this would suggest potential applications in tissue engineering and provides a tool for various clinical studies.

\section{Acknowledgements}

This research was supported by National High Technology Research and Development Program of China (2008AA02Z418), National Natural Science Foundation of China (81201378 and 81171753), Natural Science Foundation of Shanghai Science and Technology Committee (12ZR1439000) and the Young Researcher Programme of Shanghai Health Bureau (2011Y127). The funders had no role in study design, data collection and analysis, decision to publish or preparation of the manuscript.

\section{References}

1. Rees JD, Wilson AM and Wolman RL: Current concepts in the management of tendon disorders. Rheumatology (Oxford) 45: 508-521, 2006

2. Järvinen TA, Kannus P, Maffulli N and Khan KM: Achilles tendon disorders: etiology and epidemiology. Foot Ankle Clin 10: 255-266, 2005.

3. Wang JH, Iosifidis MI and Fu FH: Biomechanical basis for tendinopathy. Clin Orthop Relat Res 443: 320-332, 2006.

4. Hoffmann A and Gross G: Tendon and ligament engineering: from cell biology to in vivo application. Regen Med 1: 563-574, 2006.

5. Kuo CK, Marturano JE and Tuan RS: Novel strategies in tendon and ligament tissue engineering: Advanced biomaterials and regeneration motifs. Sports Med Arthrosc Rehabil Ther Technol 2: 20, 2010.

6. Bruder SP, Fink DJ and Caplan AI: Mesenchymal stem cells in bone development, bone repair, and skeletal regeneration therapy. J Cell Biochem 56: 283-294, 1994.

7. Jorgensen C, Gordeladze J and Noel D: Tissue engineering through autologous mesenchymal stem cells. Curr Opin Biotechnol 15: 406-410, 2004.

8. Studeny M, Marini FC, Champlin RE, Zompetta C, Fidler IJ and Andreeff M: Bone marrow-derived mesenchymal stem cells as vehicles for interferon-beta delivery into tumors. Cancer Res 62: 3603-3608, 2002.

9. Khakoo AY, Pati S, Anderson SA, et al: Human mesenchymal stem cells exert potent antitumorigenic effects in a model of Kaposi's sarcoma. J Exp Med 203: 1235-1247, 2006.

10. Wu X, Chen S, Orlando SA, et al: p85alpha regulates osteoblast differentiation by cross-talking with the MAPK pathway. J Biol Chem 286: 13512-13521, 2011.

11. Giuliani N, Bataille R, Mancini C, Lazzaretti M and Barillé S: Myeloma cells induce imbalance in the osteoprotegerin/osteoprotegerin ligand system in the human bone marrow environment. Blood 98: 3527-3533, 2001.

12. Powers CJ, McLeskey SW and Wellstein A: Fibroblast growth factors, their receptors and signaling. Endocr Relat Cancer 7: 165-197, 2000. 
13. Yu PJ, Ferrari G, Galloway AC, Mignatti P and Pintucci G: Basic fibroblast growth factor (FGF-2): the high molecular weight forms come of age. J Cell Biochem 100: 1100-1108, 2007.

14. van den Bos C, Mosca JD, Winkles J, Kerrigan L, Burgess WH and Marshak DR: Human mesenchymal stem cells respond to fibroblast growth factors. Hum Cell 10: 45-50, 1997.

15. Tsutsumi S, Shimazu A, Miyazaki K, et al: Retention of multilineage differentiation potential of mesenchymal cells during proliferation in response to FGF. Biochem Biophys Res Commun 288: 413-419, 2001.

16. Bianchi G, Banfi A, Mastrogiacomo M, et al: Ex vivo enrichment of mesenchymal cell progenitors by fibroblast growth factor 2 . Exp Cell Res 287: 98-105, 2003.

17. Baddoo M, Hill K, Wilkinson R, et al: Characterization of mesenchymal stem cells isolated from murine bone marrow by negative selection. J Cell Biochem 89: 1235-1249, 2003.

18. Choi SC, Kim SJ, Choi JH, Park CY, Shim WJ and Lim DS: Fibroblast growth factor-2 and -4 promote the proliferation of bone marrow mesenchymal stem cells by the activation of the PI3K-Akt and ERK1/2 signaling pathways. Stem Cells Dev 17: 725-736, 2008

19. Masola V,Gambaro G,TibaldiE, et al: Heparanase and syndecan-1 interplay orchestrates fibroblast growth factor-2-induced epithelial-mesenchymal transition in renal tubular cells. J Biol Chem 287: 1478-1488, 2012.

20. Solchaga LA, Penick K, Porter JD, Goldberg VM, Caplan AI and Welter JF: FGF-2 enhances the mitotic and chondrogenic potentials of human adult bone marrow-derived mesenchymal stem cells. J Cell Physiol 203: 398-409, 2005.
21. Varas L, Ohlsson LB, Honeth G, et al: Alpha10 integrin expression is up-regulated on fibroblast growth factor-2-treated mesenchymal stem cells with improved chondrogenic differentiation potential. Stem Cells Dev 16: 965-978, 2007.

22. Neubauer M, Fischbach C, Bauer-Kreisel P, et al: Basic fibroblast growth factor enhances PPARgamma ligand-induced adipogenesis of mesenchymal stem cells. FEBS Lett 577: 277-283, 2004.

23. Lai WT, Krishnappa V, Phinney DG: Fibroblast growth factor 2 (Fgf2) inhibits differentiation of mesenchymal stem cells by inducing Twist2 and Spry4, blocking extracellular regulated kinase activation, and altering Fgf receptor expression levels. Stem Cells 29: 1102-1111, 2011.

24. Frederick JP, Liberati NT, Waddell DS, Shi Y, Wang XF: Transforming growth factor beta-mediated transcriptional repression of c-myc is dependent on direct binding of Smad3 to a novel repressive Smad binding element. Mol Cell Biol 24: 2546-2559, 2004.

25. Wang L, Blouin V, Brument N, Bello-Roufai M and Francois A: Production and purification of recombinant adeno-associated vectors. Methods Mol Biol 807: 361-404, 2011.

26. Lanner $\mathrm{F}$ and Rossant J: The role of FGF/Erk signaling in pluripotent cells. Development 137: 3351-3360, 2010.

27. Ozaki K, Miyazaki S, Tanimura S and Kohno M: Efficient suppression of FGF-2-induced ERK activation by the cooperative interaction among mammalian Sprouty isoforms. J Cell Sci 118: 5861-5871, 2005. 